\title{
Zoometría de dos poblaciones de ganado Criollo de Venezuela ${ }^{1}$
}

\author{
Jorge A Ordóñez V. ${ }^{2}$ \& y Dieter Plasse $\dagger$ \\ ${ }^{2}$ Facultad de Ciencias Veterinarias - UCV, Maracay 2105 Venezuela.
}

\section{Zoometry of two Criollo cattle populations from Venezuela}

\begin{abstract}
In Venezuela, Criollo cattle, introduced by colonizers, were raised under various conditions and for different purposes. These led to the formation of two lines: the Criollo Llanero (CrLl), kept under savanna conditions, practically disappeared; and the Criollo Río Limón (CrRL) conserved in northwestern region of Zulia state, in danger of extinction. The "Cooperative Project MAC - UCV at the Los Llanos Experimental Station" included CrRL in pure and cross mating, as well as CrLl in absorption mating. Fifty CrRL foundation cows and 5o CrLl cows purchased in the Llano were the subjects of this article to describe them morphologically and compare them through statistical analysis. Some observations were eliminated due to temper that prevented taking some measurements; concluding $46 \mathrm{CrRL}$ and $43 \mathrm{CrLl}$. The $\mathrm{F}$ test was applied to distinguish the differences between the lines of each measure. The Line effect was significant $(\mathrm{p}<0.05)$ or highly significant $(\mathrm{p}<0.01)$ for 21 of 24 variables. CrRL outperformed CrLl in the relevant dimensions. The Weight in CrRL was $20 \%$ higher than in CrLl (p< 0.01). In the other dimensions, the advantage ranged between 3 and $8 \%$, in favor of CrRL. The correlations between relevant variables were positive and significant $(\mathrm{p}<0.01)$, always higher in CrRL. The few negative sign correlations were not significant $(\mathrm{p}>0.05)$. Fifty years after the observations were made, time altered the research justification, from to generate basic knowledge, today its relevance lies in the valuation of autochthonous germplasm and its contribution to the genetic variability necessary to couple specific genotypes with particular breeding systems and altered environmental conditions. The decline of these populations reported in the literature reveals that it might be late to recommend their conservation and assessment and that the initiatives for their preservation and use have been insufficient and ineffective.
\end{abstract}

Key words: Calabozo, cows, extinction, genetic resources, Limonero

Resumen. En Venezuela, el ganado criollo, introducido por los colonizadores, fue criado bajo condiciones diversas y, con diferentes propósitos. Ello indujo la formación de dos líneas: el Criollo Llanero (CrLl), mantenido bajo condiciones de sábana, prácticamente desaparecido; y el Criollo Río Limón (CrRL) conservado en el noroeste del estado Zulia, en peligro de extinción. El "Proyecto Cooperativo MAC - UCV en la Estación Experimental de Los Llanos" incluyó CrRL en cría pura y cruzamiento, así como CrLl en apareamiento de absorción. Cincuenta vacas CrRL de fundación y 50 vacas CrLl compradas en el Llano fueron el sujeto de este trabajo con el objetivo de describirlas morfológicamente y compararlas mediante análisis estadístico. Se eliminaron observaciones debido al temperamento que impidió tomar algunas medidas; concluyendo $46 \mathrm{CrRL}$ y $43 \mathrm{CrLl}$. Se aplicó la prueba $\mathrm{F}$ para distinguir las diferencias entre líneas de cada medida. Línea resultó significativo $(\mathrm{p}<0.05)$ o altamente significativo ( $\mathrm{p}<0.01)$ para 21 de 24 variables. CrRL superó a CrLl en las dimensiones relevantes. El Peso en CrRL fue $20 \%$ mayor que en CrLl $(\mathrm{p}<$ o.01). En las demás dimensiones, la ventaja osciló entre 3 y 8 \%, a favor de CrRL. Las correlaciones entre variables relevantes resultaron positivas y significativas $(\mathrm{p}<0.01)$, siempre mayores en CrRL. Las pocas correlaciones de signo negativo resultaron no significativas $(\mathrm{p}>0.05)$. Cincuenta años después de realizadas las observaciones reportadas, el tiempo alteró la justificación; y de generar información básica, hoy su relevancia radica en la valoración del germoplasma autóctono y su contribución a la variabilidad genética necesaria para acoplar genotipos específicos a sistemas de cría particulares y condiciones ambientales alteradas. La declinación de estas poblaciones reportada en la literatura evidencia que podría ser tarde para recomendar su conservación y valoración y que las iniciativas para la preservación y aprovechamiento de ambas poblaciones han resultado insuficientes e inefectivas.

Palabras clave: Calabozo, extinción, Limonero, recursos genéticos, vacas

Recibido: 2020-07-27. Aceptado: 2020-11-06

${ }^{1}$ Este trabajo se deriva de una Tesis requisito parcial para obtener la credencial de Preparador de la Catedra de Genética, mayo 1970 y fue realizada en las instalaciones de la Estación Experimental de los Llanos - Calabozo, Venezuela, Ministerio de Agricultura y Cría.

22Autor para la correspondencia: jaordonezv@gmail.com 


\section{Introducción}

En Venezuela, la mayoría de las poblaciones bovinas locales, y en particular, las criollas, están amenazadas por el cruzamiento indiscriminado o la insostenibilidad de los sistemas de producción (Bracho et al., 2002). Los caracteres importantes que se han atribuido a las razas locales son: la resistencia a las enfermedades, la tolerancia a la alimentación de baja calidad, el rendimiento reproductivo relativamente superior, especialmente en condiciones ambientales adversas, la tolerancia a temperaturas extremas, y los bajos requerimientos de mantenimiento (Bottani, 2020). La conservación de las razas criollas y su valoración contribuye a preservar la variación genética necesaria para acoplar genotipos específicos con particulares sistemas de cría y condiciones ambientales alteradas tal cómo el cambio climático, enfermedades infecciosas emergentes, desarrollo de mercados y preferencias del consumidor (Ordóñez, 2020). Además, las razas autóctonas constituyen un bien cultural, referencia en las tradiciones locales que desempeñan un papel en la vida social de las poblaciones rurales (Gandini y Villa, 2003 citado por Bottani (2020)). Sin embargo, el uso de animales criollos en programas de cría estructurados es prácticamente inexistente, ante todo porque la comparación entre decisiones sobre el uso de recursos productivos de la finca se ha basado en obtener la máxima producción por animal cuando la meta debe ser el desempeño económico: utilidad y rentabilidad (Madalena, 2002). La ventaja de uno u otro genotipo animal depende del ambiente proporcionado. Ello implica aumentar su conocimiento, definir su importancia y utilizarlos eficientemente (Bottani, 2020).

El ganado criollo venezolano, originario del cruzamiento desordenado de animales introducidos por los conquistadores europeos durante la colonización de América (Rouse, 1977), fue mantenido bajo condiciones diversas y utilizados con diferentes propósitos, lo cual indujo a la formación de por lo menos dos líneas de ganado criollo (Bodisco y Abreu, 1981): el ganado Criollo Llanero (CrLl), mantenido bajo condiciones de sabana, en cría extensiva y explotado para la producción de carne (RodríguezViso, s/f) y el ganado Criollo Río Limón o Criollo Limonero (CrRL) conservado y perfeccionado por los métodos empíricos en la región de Río Limón (distritos Mara y Páez) del estado Zulia, en Venezuela, descrito por Ríos et al. (1959), citado por Bodisco y Abreu (1981). Luego de la introducción del cebú y las razas europeas exóticas ambas poblaciones se redujeron ostensiblemente. Florio-Luis y Pineda-Graterol (2018) consideran al CrRL en peligro de extinción. Por su parte el CrLl se encuentra prácticamente desaparecido. Promover la protección y valoración del recurso genético pasa por completar su inventario y caracterización, fomentar su utilización sustentable e impulsar su valoración. El desconocimiento que de este biotipo se tiene y la necesidad no solo económica sino también zoológica e histórica, hizo perentoria su descripción.

En el año 1965, se inició un programa de apareamiento en la Estación Experimental de los Llanos, Calabozo, Estado Guárico, Venezuela (Plasse, 1981). Las vacas CrRL de fundación fueron transferidas desde un proyecto de Criollo lechero en Maracay (Abreu et al., 1977) citado por Plasse (1981) habiendo sido descartadas por causas que no imposibilitaron su empleo para la producción de carne. Las CrLl se compraron en diversas zonas del Llano venezolano (Plasse, 1981). Esos rebaños de fundación fueron el sujeto de este trabajo con la finalidad de generar información básica a ser utilizada en el desarrollo del programa de cruzamiento y selección de ganado de carne que tuvo lugar en la Estación Experimental de Los Llanos, a partir de 1965. La conformación corporal en los animales se considera un carácter subjetivo, pero las mediciones corporales concretas y su variación normal en una determinada población permite cuantificar la conformación corporal y diferenciar objetivamente las poblaciones (Cevallos, 2012). El objetivo de este estudio consistió en describir morfológicamente y comparar mediante análisis estadístico dos rebaños de vacas adultas de ganado criollo de diferente procedencia.

\section{Materiales y métodos}

La Estación Experimental de los Llanos está ubicada en los Bancos de San Pedro en el kilómetro 28 de la carretera Calabozo - San Fernando de Apure $\left(8^{\circ} 44^{\prime} 51.9^{\prime \prime} \mathrm{N} 67^{\circ} 32^{\prime} 03.8^{\prime \prime W}\right)$, a 83 m.s.n.m. en el centro del Sistema de Riego Río Guárico. Establecida en 1958, para la época de la recolección de los datos del presente trabajo era administrada por el Fondo Nacional de Investigaciones Agropecuarias
(FONAIAP), adscrito al Ministerio de Agricultura y Cría, hoy se denomina Centro de Investigaciones Agropecuarias del Estado Guárico (CIAE Guárico) del Instituto Nacional de Investigaciones Agrícolas (INIA), adscrito al Ministerio de Ciencia y Tecnología. A partir de 1966, allí se desarrolló el "Proyecto Cooperativo MAC - UCV de investigación con ganado de carne en la Estación Experimental de Los Llanos", cuyo plan de 
apareamiento incluía vacas CrRL en cría pura y en cruzamiento de absorción con Brahman y Santa Gertrudis y cruzamiento alterno con Brahman; y vacas CrLl en cruzamiento de absorción con Criollo Río Limón y Brahman.

Las vacas de fundación según Plasse, (1981), tanto CrRL como CrLl eran ejemplares típicos de los usados contemporáneamente en la ganadería venezolana para la producción de carne, y en sistemas de doble propósito carne-leche. Los diferentes grupos raciales permanecieron juntos en sabana natural con riego durante la temporada seca; sin embargo, la sabana de la Estación Experimental no estaba en condiciones óptimas y el estado nutricional del rebaño nunca fue satisfactorio. El manejo estuvo subordinado a una temporada de monta limitada de cuatro meses, durante la estación seca. Durante la temporada de monta se conformaron los grupos de apareamiento constituidos por 25 vacas y 2 toros. Los resultados de la primera generación, es decir, los correspondientes a las vacas de fundación y sus hijos puros y F1, fueron analizados y publicados en una serie de trabajos (Frómeta et al., 1974; Linares et al., 1974a, b; Peña de Borsotti et al., 1974; Ordóñez et al., 1974; Plasse et al., 1974a, b).

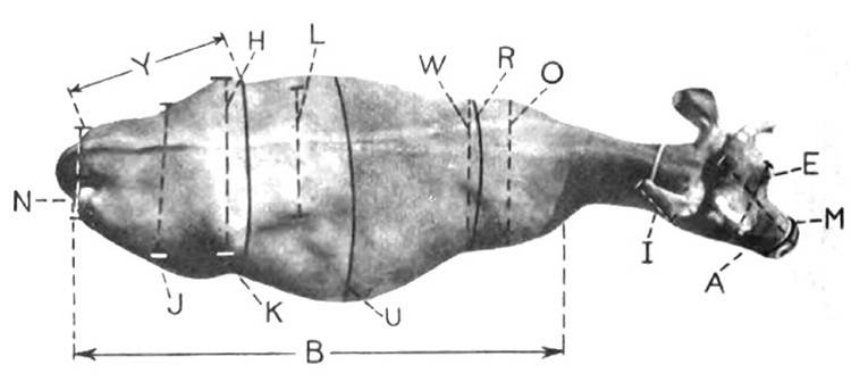

Figura 1. Identificación de las medidas - Vista dorsal. Fuente: Lush y Copeland (1930).

Las medidas analizadas y su comparación entre líneas incluyen: A. Longitud de la cabeza (LnCa), B. Longitud del cuerpo (LnCp), C. Alzada a la rodilla (AlRd), D. Profundidad del pecho (PfPc), E. Ancho de la cabeza (AnCa), F. Profundidad mayor al esternón (PmyE), G. Profundidad mínima al esternón (PmnE), H. Ancho de la grupa (AnGr), I. Longitud de la oreja (LnOj), N. Ancho de la pelvis (AnPv), O. Ancho de la espalda (AnEs), P. Alzada a la cruz (AlCz), R. Perímetro cardíaco (PrCr), S. Espesor de la piel doblada (EsPd), T. Alzada a la grupa (AlGr), U. Perímetro abdominal (PrAb), V. Perímetro de la caña (PrCñ), W. Ancho del pecho (AnPc), Y. Longitud de la pelvis ( $\mathrm{LnPv}$ ), Z Alzada al codo (AlCd). Además, Peso, Alzada a la base de la cola (AlBC), Longitud del cuerno ( $\mathrm{LnCn}$ ), y
Los muestreos se realizaron sobre vacas adultas multíparas. La edad al momento de la compra fue estimada mediante cronometría dentaria en el caso del CrLl por lo que fue desestimada para los efectos de análisis. Se trabajó un total de 100 animales, 50 de cada raza. Del total de observaciones se eliminaron vacas por tener datos incompletos, mayormente $\mathrm{CrLl}$, debido a mala disposición que impidió tomar algunas medidas relevantes; concluyendo el proyecto $46 \mathrm{CrRL}$ y $43 \mathrm{CrLl}$. También se eliminaron aquellas medidas correspondientes a la ubre, por su poca exactitud. Cada vaca lactante fue evaluada durante el primer mes post parto, entre noviembre de 1968 y marzo de 1969. En febrero de 1969 se midieron las vacas secas, estando vacías ambos grupos pues las evaluaciones se realizaron antes del inicio de la temporada de monta de 1969. Las medidas se realizaron solo una vez puesto que Lush y Copeland (1930) encontraron que las mediciones únicas fueron lo suficientemente precisas. Las mediciones de alzada se realizaron con un bastón zoométrico, las anchuras con un pie de rey, los perímetros con cinta métrica inextensible y el espesor de la piel doblada con vernier. Se utilizó el método descrito por Lush y Copeland (1930) tal como se muestra en las figuras 1 y 2.

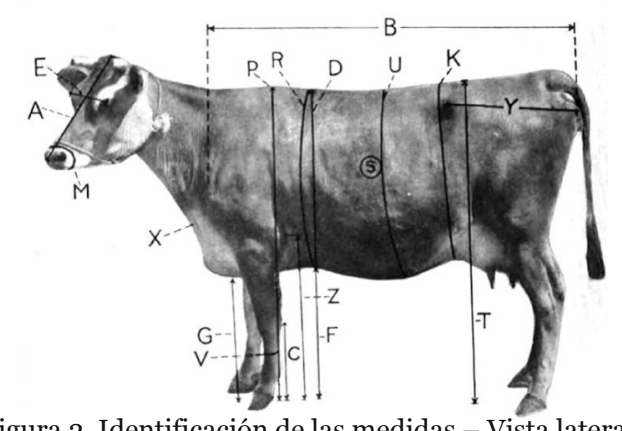

Figura 2. Identificación de las medidas - Vista lateral. Fuente: Lush y Copeland (1930).

Perímetro del cuerno en la base (PrCB), que no se muestran en las Figuras 1 y 2.

Para obtener los estadísticos descriptivos y completar los análisis de varianza se utilizó una calculadora electrónica FACIT serie 1120 (1966), máquina capaz de calcular automáticamente la raíz cuadrada de un número de 18 dígitos. Se aplicó la prueba de F descrita por Fisher (1925), a cada variable para distinguir las diferencias entre Líneas o Procedencias. La significancia estadística se probó comparando el estadístico $\mathrm{F}=\mathrm{CM}_{\text {Línea }} / \mathrm{CM}_{\text {residual }}$ calculado con el valor crítico de $\mathrm{F}$ determinado a partir de tablas, en función de los grados de libertad del numerador y del denominador y del nivel de significancia. 


\section{Resultados y Discusión}

Las variables, el número de observaciones, sus promedios y desviaciones típicas se señalan en el cuadro 1, por línea y para el material en conjunto.

Cuadro 1. Variables, número de observaciones (n), promedios $(\overline{\mathrm{X}})$ y desviaciones típicas (S) por línea y para el material en conjunto.

\begin{tabular}{|c|c|c|c|c|c|c|c|c|c|}
\hline \multirow[t]{2}{*}{ Variables } & \multicolumn{3}{|c|}{$\begin{array}{c}\text { Criollo } \\
\text { Río Limón } \\
\end{array}$} & \multicolumn{3}{|c|}{$\begin{array}{c}\text { Criollo } \\
\text { Llanero } \\
\end{array}$} & \multicolumn{3}{|c|}{ Total } \\
\hline & $\mathrm{n}$ & $\bar{X}$ & $\mathrm{~S}$ & $\mathrm{n}$ & $\bar{X}$ & $\mathrm{~S}$ & $\mathrm{n}$ & $\bar{X}$ & $\mathrm{~S}$ \\
\hline Peso (kg) & 46 & 371.0 & 40.9 & 43 & 308.3 & 30.9 & 89 & 340.7 & 48.0 \\
\hline $\mathrm{AlCz}(\mathrm{cm})$ & 46 & 123.2 & $4 \cdot 4$ & 43 & 117.4 & $3 \cdot 5$ & 89 & 120.4 & 4.9 \\
\hline $\operatorname{AlGr}(\mathrm{cm})$ & 46 & 126.7 & 4.9 & 43 & 122.2 & 4.8 & 89 & 124.6 & $5 \cdot 3$ \\
\hline $\mathrm{AlBC}(\mathrm{cm})$ & 46 & 127.9 & 4.5 & 43 & 122.2 & 5.1 & 89 & 125.1 & 5.6 \\
\hline $\operatorname{PfPc}(\mathrm{cm})$ & 46 & 66.4 & 2.7 & 43 & 62.7 & 2.1 & 89 & 64.6 & 3.0 \\
\hline PmyE (cm) & 46 & 58.6 & 4.6 & 43 & 56.3 & 5.2 & 89 & 57.2 & 5.0 \\
\hline PmnE (cm) & 46 & 50.5 & 4.5 & 43 & 51.9 & 7.2 & 89 & 51.2 & 6.0 \\
\hline $\mathrm{AlCd}(\mathrm{cm})$ & 46 & 71.4 & 4.2 & 43 & 68.2 & 4.7 & 89 & 69.9 & $4 \cdot 7$ \\
\hline AlRd (cm) & 46 & 36.9 & 2.8 & 43 & 35.1 & 4.4 & 89 & 36.0 & 3.8 \\
\hline $\mathrm{LnCp}(\mathrm{cm})$ & 46 & 147.1 & 4.9 & 43 & 138.6 & 5.0 & 89 & 143.0 & 6.5 \\
\hline $\mathrm{LnCa}(\mathrm{cm})$ & 46 & 51.1 & 1.7 & 43 & 49.4 & 1.9 & 89 & 50.3 & 2.0 \\
\hline $\mathrm{LnPv}(\mathrm{cm})$ & 46 & 50.8 & 2.2 & 43 & $47 \cdot 3$ & 1.9 & 89 & 49.1 & 2.7 \\
\hline $\mathrm{AnCa}(\mathrm{cm})$ & 46 & 21.9 & 0.95 & 43 & 21.0 & 0.9 & 89 & 21.5 & 1.0 \\
\hline $\operatorname{AnPv}(\mathrm{cm})$ & 46 & 27.8 & 3.0 & 43 & 26.2 & 2.8 & 89 & 27.1 & 3.0 \\
\hline $\operatorname{AnGr}(\mathrm{cm})$ & 46 & 49.2 & 2.6 & 43 & 45.2 & 2.6 & 89 & $47 \cdot 3$ & $3 \cdot 3$ \\
\hline AnEs (cm) & 46 & $35 \cdot 5$ & 3.5 & 43 & 33.6 & $3 \cdot 3$ & 89 & 34.6 & 3.5 \\
\hline $\operatorname{AnPc}(\mathrm{cm})$ & 46 & 30.5 & 3.5 & 43 & 28.8 & 3.6 & 89 & 29.7 & 3.6 \\
\hline $\operatorname{EsPd}(\mathrm{mm})$ & 46 & 14.0 & 0.3 & 43 & 12.3 & 2.2 & 89 & 13.2 & 2.6 \\
\hline $\operatorname{PrCñ~}(\mathrm{cm})$ & 46 & 17.2 & 1.3 & 43 & 15.9 & 1.4 & 89 & 16.6 & 1.5 \\
\hline $\operatorname{PrCr}(\mathrm{cm})$ & 46 & 172.1 & $7 \cdot 4$ & 43 & 164.6 & 7.1 & 89 & 168.5 & 8.1 \\
\hline $\operatorname{PrAb}(\mathrm{cm})$ & 43 & 198.0 & 10.8 & 43 & 192.3 & 10.9 & 89 & 194.2 & 11.5 \\
\hline $\operatorname{LnCn}(\mathrm{cm})$ & 32 & 36.5 & 6.5 & 41 & 42.3 & $5 \cdot 3$ & 73 & 39.8 & 6.5 \\
\hline $\operatorname{PrCB}(\mathrm{cm})$ & 32 & 18.2 & 1.6 & 42 & 17.7 & 1.9 & 74 & 17.9 & 1.7 \\
\hline $\mathrm{LnOj}(\mathrm{cm})$ & 45 & 16.1 & 0.9 & 36 & 16.6 & 1.41 & 81 & 16.3 & 1.1 \\
\hline
\end{tabular}

AlCz: Alzada a la cruz. AlGr: Alzada a la grupa. AlBC: Peso, Alzada a la base de la cola. PfPc: Profundidad del pecho. PmyE: Profundidad mayor al esternón. PmnE: Profundidad mínima al esternón. AlCd: Alzada al codo. AlRd: Alzada a la rodilla. LnCp: Longitud del cuerpo. LnCa: Longitud de la cabeza. LnPv: Longitud de la pelvis. AnCa: Ancho de la cabeza. AnPv: Ancho de la pelvis. AlGr: Alzada a la grupa. AnEs: Ancho de la espalda. AnPc: Ancho del pecho. EsPd: Espesor de la piel doblada. PrCñ: Perímetro de la caña. PrCr: Perímetro cardíaco. PrAb: Perímetro abdominal. LnCn: Longitud del cuerno. PrCB: Perímetro del cuerno en la base. LnOj: Longitud de la oreja.

La influencia de los factores ambientales sobre el peso de las vacas no fue considerada en este trabajo. El único peso realizado coincidió con la transición entre las estaciones lluviosa y seca (noviembre a febrero) lo cual hace presumir que se encontraban en buena condición corporal. La influencia de la estacionalidad y su efecto sobre el peso de las vacas adultas fue demostrada por Bodisco y Pacheco (1962) quienes obtuvieron en vacas adultas CrRL. promedio de $391 \mathrm{~kg}$ en el período seco, significativamente inferior a los 404 $\mathrm{kg}$ en el período lluvioso.

Los valores de desviación típica y los coeficientes de variación (CV) obtenidos (no mostrados) indican que en CrRL la media de CV apenas alcanzó el 6.7 \%. Sólo 4 de 24 variables superaron $10 \%$ de CV sin alcanzar el $20 \%$, poniendo en evidencia la escasa variabilidad de la población; mientras que el CrLl resultó ligeramente más variable, pues 8 de 24 variables superaron el $10 \%$ de CV, destacándose el Espesor de la piel doblada cuyo CV alcanzó el 18 \%. Estos resultados contrastan con los obtenidos por Cevallos et al. (2016) quienes reportaron "discreta uniformidad zoométrica" en bovinos criollos de Manabí. Ecuador con CV inferiores a $20 \%$; señalando además que la homogeneidad fenotípica observada de variables zoométricas fue sensiblemente menor que otras razas criollas iberoamericanas incluido el CrRL de Venezuela.

En el cuadro 2 se resumen los análisis de varianza de cada variable. El efecto de Línea resultó significativo o altamente significativo para todas las variables excepto Profundidad mínima al esternón, Longitud de la oreja y Perímetro del cuerno en la base. 
Cuadro 2. Análisis de varianza de las variables: fuentes de variación, grados de libertad (G.L.) y cuadrados medios (C.M.).

\begin{tabular}{|c|c|c|c|}
\hline Variables & Fuentes de variación & G. L. & C. M. \\
\hline \multirow[t]{2}{*}{ Peso (kg) } & Línea & 1 & $87297.00^{* * *}$ \\
\hline & Residual & 87 & 1326.10 \\
\hline \multirow[t]{2}{*}{$\mathrm{AlCz}(\mathrm{cm})$} & Línea & 1 & $727.90^{* *}$ \\
\hline & Residual & 87 & 16.14 \\
\hline \multirow[t]{2}{*}{$\operatorname{AlGr}(\mathrm{cm})$} & Línea & 1 & $449.60^{* *}$ \\
\hline & Residual & 87 & 23.76 \\
\hline \multirow[t]{2}{*}{$\operatorname{AlBC}(\mathrm{cm})$} & Línea & 1 & $712.60^{* *}$ \\
\hline & Residual & 87 & 23.08 \\
\hline \multirow[t]{2}{*}{$\operatorname{PfPc}(\mathrm{cm})$} & Línea & 1 & $312.51^{* *}$ \\
\hline & Residual & 87 & 5.73 \\
\hline \multirow[t]{2}{*}{$\operatorname{PmyE}(\mathrm{cm})$} & Línea & 1 & $116.74^{*}$ \\
\hline & Residual & 87 & 24.40 \\
\hline \multirow[t]{2}{*}{$\operatorname{PmnE}(\mathrm{cm})$} & Línea & 1 & 42.58 \\
\hline & Residual & 87 & 35.85 \\
\hline \multirow[t]{2}{*}{$\mathrm{AlCd}(\mathrm{cm})$} & Línea & 1 & $244.80^{* *}$ \\
\hline & Residual & 87 & 19.82 \\
\hline \multirow[t]{2}{*}{$\operatorname{AlRd}(\mathrm{cm})$} & Línea & 1 & $79.28^{*}$ \\
\hline & Residual & 87 & 13.46 \\
\hline \multirow[t]{2}{*}{$\operatorname{LnCp}(\mathrm{cm})$} & Línea & 1 & $1624.28^{* *}$ \\
\hline & Residual & 87 & 24.63 \\
\hline \multirow[t]{2}{*}{$\mathrm{LnCa}(\mathrm{cm})$} & Línea & 1 & $62.69^{* * *}$ \\
\hline & Residual & 87 & 3.20 \\
\hline \multirow[t]{2}{*}{$\operatorname{LnPv}(\mathrm{cm})$} & Línea & 1 & $325.01^{* *}$ \\
\hline & Residual & 87 & 6.04 \\
\hline \multirow[t]{2}{*}{$\mathrm{AnCa}(\mathrm{cm})$} & Línea & 1 & $19.00^{* *}$ \\
\hline & Residual & 87 & 0.89 \\
\hline \multirow[t]{2}{*}{$\operatorname{AnPv}(\mathrm{cm})$} & Línea & 1 & $62.23^{* *}$ \\
\hline & Residual & 87 & 8.36 \\
\hline \multirow[t]{2}{*}{$\operatorname{AnGr}(\mathrm{cm})$} & Línea & 1 & $347.96^{* *}$ \\
\hline & Residual & 87 & $7 \cdot 36$ \\
\hline \multirow[t]{2}{*}{ AnEs $(\mathrm{cm})$} & Línea & 1 & $79.77^{* *}$ \\
\hline & Residual & 87 & 11.60 \\
\hline \multirow[t]{2}{*}{$\operatorname{AnPc}(\mathrm{cm})$} & Línea & 1 & $66.47^{*}$ \\
\hline & Residual & 87 & 12.34 \\
\hline \multirow[t]{2}{*}{$\operatorname{EsPd}(\mathrm{cm})$} & Línea & 1 & $63.79^{* * *}$ \\
\hline & Residual & 87 & 5.88 \\
\hline \multirow[t]{2}{*}{$\operatorname{PrCñ}(\mathrm{cm})$} & Línea & 1 & $37.44^{* *}$ \\
\hline & Residual & 87 & 1.78 \\
\hline \multirow[t]{2}{*}{$\operatorname{PrCr}(\mathrm{cm})$} & Línea & 1 & $1274.81^{* *}$ \\
\hline & Residual & 87 & 40.90 \\
\hline \multirow[t]{2}{*}{$\operatorname{PrAb}(\mathrm{cm})$} & Línea & 1 & $1318.04^{* *}$ \\
\hline & Residual & 87 & 117.63 \\
\hline \multirow[t]{2}{*}{$\operatorname{LnCn}(\mathrm{cm})$} & Línea & 1 & $600.93^{* *}$ \\
\hline & Residual & 71 & 34.49 \\
\hline \multirow[t]{2}{*}{$\operatorname{PrCB}(\mathrm{cm})$} & Línea & 1 & 3.67 \\
\hline & Residual & 72 & 3.13 \\
\hline $\operatorname{LnOj}(\mathrm{cm})$ & Línea & 1 & 5.11 \\
\hline & Residual & 79 & 1.32 \\
\hline
\end{tabular}

AlCz: Alzada a la cruz. AlGr: Alzada a la grupa. AlBC: Peso, Alzada a la base de la cola. PfPc: Profundidad del pecho. PmyE: Profundidad mayor al esternón. PmnE: Profundidad mínima al esternón. AlCd: Alzada al codo. AlRd: Alzada a la rodilla. LnCp: Longitud del cuerpo. LnCa: Longitud de la cabeza. LnPv: Longitud de la pelvis. AnCa: Ancho de la cabeza. AnPv: Ancho de la pelvis. AlGr: Alzada a la grupa. AnEs: Ancho de la espalda. AnPc: Ancho del pecho. EsPd: Espesor de la piel doblada. PrCñ: Perímetro de la caña. PrCr: Perímetro cardíaco. PrAb: Perímetro abdominal. LnCn: Longitud del cuerno. PrCB: Perímetro del cuerno en la base. LnOj: Longitud de la oreja.

${ }^{*} \mathrm{p}<0.05 .{ }^{* *} \mathrm{p}<0.01$ 
En el Cuadro 3 se observa como el ganado CrRL superó al $\mathrm{CrLl}$ en las dimensiones más importantes expresado como Superioridad $\mathrm{CrRL}=(\overline{\mathrm{X}} \mathrm{CrRL} / \overline{\mathrm{X}} \mathrm{CrLl}) \times$ 100. El Peso en el CrRL fue $20 \%$ mayor que en el CrLl. Para las demás dimensiones, la ventaja osciló entre 3 y $8 \%$ a favor del CrRL, tal como se muestra en el Cuadro 3. Es posible que el manejo y la selección bajo un sistema de explotación lechera durante la formación de las líneas sean responsables de esta ventaja.

En su extenso trabajo "Producción de leche por vacas criollas puras" Bodisco y Abreu (1977) resumieron las observaciones bovino-métricas del Ganado Criollo Latinoamericano disponibles hasta la fecha. Las medidas del ganado Caracú en Brasil fueron publicadas por Jordão (1949; 1956), las del Blanco orejinegro (BON) en Colombia por Gracia (1947); Ospina (1950) y Botero (1976), las del Costeño Con Cuernos (CCC) igualmente en Colombia por Pinzón (1952) y Rubio (1976) y las del CrRL en Venezuela por Ríos et al. (1959); Bodisco y Pacheco (1962); Magofke y Bodisco (1966) y más recientemente por Contreras et

Cuadro 3. Diferencias entre Criollo Río Limón y Criollo Llanero (\%). al. (2011). En la sierra media-alta en la cordillera de los Andes del Sur del Ecuador, Aguirre et al. (2011) describieron tres poblaciones: el Negro Lojano de la Sierra Sur, el Encerado y el Cajamarca; mientras que Cevallos et al. (2016) realizaron la caracterización morfométrica del bovino criollo en la provincia de Manabí. Por otra parte. Bottani (2020) en Bolivia describió tres poblaciones de bovinos criollos: el Chaqueño, localizado en el sureste de Bolivia, está adaptado a un ambiente de bosque seco y criado para la producción de carne; el Saavedreño (CEASIP) adaptado a una llanura tropical y destinado a la producción de carne y una población de ganado criollo de Pasorapa (PASO), bien adaptada a un ecosistema xerófito y dieta en base a residuos de cultivos y el consumo de especies vegetales nativas. Canales (2014) completó su tesis doctoral caracterizando la variabilidad morfométrica y genética de un hato Criollo Lechero Tropical en Otapa, Veracruz. Finalmente, Martínez (2008) hizo lo propio con el Criollo Patagónico argentino.

\begin{tabular}{|c|c|c|c|}
\hline & \multicolumn{3}{|c|}{$($ Criollo Llanero $=100)$} \\
\hline Variables & Criollo Río Limón & Variables & Criollo Río Limón \\
\hline Peso (kg) & 120.3 & AnCa (cm) & $\overline{104 \cdot 3}$ \\
\hline $\mathrm{AlCz}(\mathrm{cm})$ & 104.9 & $\operatorname{AnPv}(\mathrm{cm})$ & 106.1 \\
\hline $\operatorname{AlGr}(\mathrm{cm})$ & 103.7 & $\operatorname{AnGr}(\mathrm{cm})$ & 108.8 \\
\hline $\operatorname{AlBC}(\mathrm{cm})$ & 104.7 & AnEs $(\mathrm{cm})$ & 105.7 \\
\hline $\operatorname{PfPc}(\mathrm{cm})$ & 105.9 & $\operatorname{AnPc}(\mathrm{cm})$ & 105.9 \\
\hline PmyE $(\mathrm{cm})$ & 104.1 & EsPd $(\mathrm{cm})$ & 113.8 \\
\hline PmnE (cm) & $97 \cdot 3$ & $\operatorname{PrCñ~}(\mathrm{cm})$ & 108.2 \\
\hline $\mathrm{AlCd}(\mathrm{cm})$ & 104.7 & $\operatorname{PrCr}(\mathrm{cm})$ & 104.6 \\
\hline AlRd (cm) & 105.1 & $\operatorname{PrAb}(\mathrm{cm})$ & 103.0 \\
\hline $\operatorname{LnCp}(\mathrm{cm})$ & 106.1 & $\operatorname{LnCn}(\mathrm{cm})$ & 86.3 \\
\hline $\mathrm{LnCa}(\mathrm{cm})$ & 103.4 & $\operatorname{PrCB}(\mathrm{cm})$ & 102.8 \\
\hline $\mathrm{LnPv}(\mathrm{cm})$ & 107.4 & $\mathrm{LnOj}(\mathrm{cm})$ & 97.0 \\
\hline
\end{tabular}

AlCz: Alzada a la cruz. AlGr: Alzada a la grupa. AlBC: Peso, Alzada a la base de la cola. PfPc: Profundidad del pecho. PmyE: Profundidad mayor al esternón. PmnE: Profundidad mínima al esternón. AlCd: Alzada al codo. AlRd: Alzada a la rodilla. LnCp: Longitud del cuerpo. LnCa: Longitud de la cabeza. LnPv: Longitud de la pelvis. AnCa: Ancho de la cabeza. AnPv: Ancho de la pelvis. AlGr: Alzada a la grupa. AnEs: Ancho de la espalda. AnPc: Ancho del pecho. EsPd: Espesor de la piel doblada. PrCñ: Perímetro de la caña. PrCr: Perímetro cardíaco. PrAb: Perímetro abdominal. LnCn: Longitud del cuerno. PrCB: Perímetro del cuerno en la base. LnOj: Longitud de la oreja.

El Cuadro 4 muestra de manera apretada 16 denominaciones de poblaciones Latinoamericanas analizadas por 18 trabajos publicados entre 1949 y 2020, muchas otras quedaron fuera. En él se resume 10 de las 24 medidas disponibles, limitándose a aquellas con 10 o más reportes en la literatura revisada, que suponemos las de mayor interés. A primera vista sorprende la similitud entre poblaciones, siendo comparables las diferencias entre observaciones de una misma raza con aquellas entre razas.
Aunque todos los animales trabajados fueron vacas adultas, comparar resultados obtenidos en vacas de edad o número de parto, estado de lactación o de preñez, estación, dieta y manejo distintos, realizado por equipos de investigadores en lugares, instalaciones, métodos e instrumentos también diferentes, luce aventurado por decir lo menos.

A riesgo de caer en lugares comunes, los Criollos son en general animales pequeños, con excepción del Caracú, de mayor envergadura, mientras que el Negro 
Lojano, el Encerado y el Cajamarca ecuatorianos, resultaron particularmente cortos y livianos. La contextura estrecha y delgada de los diferentes biotipos justifica la generalizada apreciación de que aproximan a tipos lecheros. El Criollo Lechero Tropical
(CLT) mostró cabeza pequeña y pelvis corta en relación con las demás razas. La escasa estatura y bajo peso del BON corresponde a los animales disponibles entre 1947 y 1950, fecha en que se realizaron los trabajos.

Cuadro 4. Medias de las principales medidas realizadas en diferentes poblaciones de Criollo en América Latina

\begin{tabular}{|c|c|c|c|c|c|c|c|c|c|c|c|c|c|}
\hline \multicolumn{2}{|c|}{ Denominación* País } & Referencias** & \multirow{2}{*}{$\begin{array}{c}\begin{array}{c}\text { Alzada } \\
\text { a }\end{array} \\
\text { la cruz } \\
(\mathrm{cm})\end{array}$} & \multirow{2}{*}{$\begin{array}{c}\begin{array}{c}\text { Longitud } \\
\text { del } \\
\text { cuerpo } \\
(\mathrm{cm})\end{array} \\
147\end{array}$} & \multirow{2}{*}{$\begin{array}{c}\begin{array}{c}\text { Alzada } \\
\text { a la } \\
\text { grupa } \\
(\mathrm{cm})\end{array} \\
127\end{array}$} & \multirow{2}{*}{$\begin{array}{c}\begin{array}{c}\text { Ancho } \\
\text { de la } \\
\text { cabeza } \\
(\mathrm{cm})\end{array} \\
22\end{array}$} & \multirow{2}{*}{ 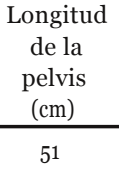 } & \multirow{2}{*}{$\begin{array}{c}\begin{array}{c}\text { Perímetro } \\
\text { cardiaco }\end{array} \\
(\mathrm{cm}) \\
172\end{array}$} & \multirow{2}{*}{$\begin{array}{c}\begin{array}{c}\text { Profundidad } \\
\text { del } \\
\text { pecho } \\
(\mathrm{cm})\end{array} \\
66\end{array}$} & \multirow{2}{*}{ 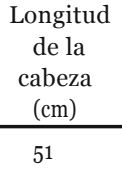 } & \multirow{2}{*}{$\begin{array}{l}\text { Perímetro } \\
\text { de la } \\
\text { caña } \\
(\mathrm{cm})\end{array}$} & \multicolumn{2}{|c|}{$\begin{array}{r}\text { Peso } \\
(\mathrm{kg})\end{array}$} \\
\hline CrRL & Venezuela & & & & & & & & & & & 371 & \\
\hline CrLl & Venezuela & & 117 & 139 & 122 & 21 & 47 & 165 & 63 & 49 & 16 & 308 & \\
\hline CrRL & Venezuela & 1) 2) 12) 18) & 122.124 & 143.130 & 128.130 & 23 & 46.38 & 165.174 & 64 & $47 \cdot 49$ & 16. 18 & 406. & 3. 477.404 \\
\hline Caracú & Brasil & 3) 8) & 128 & 147 & & & 44 & 187 & 67 & & 18 & & 522 \\
\hline BON & Colombia & 4) 9) 10) & & 142 & 122 & & & 169 & 63 & & 17 & & 359.360 \\
\hline $\begin{array}{l}\text { CCC } \\
\text { Caracú }\end{array}$ & Colombia & 5) 7) & 123 & 152 & & & & 169 & & & & & 380 \\
\hline Caldeano & Colombia & 6) & 135 & & & & & & & & & & \\
\hline Cr Pasorapa & Bolivia & 13） & 122 & & 129 & & & & & & & & \\
\hline Cr Chaco & Bolivia & 13) & 122 & & 127 & & & 167 & & & & & \\
\hline Cr CEASIP & Bolivia & 13） & 127 & & 137 & & 37 & & 63 & & & & \\
\hline Cr Negro jano & & Ecuador 14） & & 125 & 134 & & 22 & 41 & 67 & 48 & 18 & & 300 \\
\hline Cr Encerado & Ecuador & 14) & 119 & 133 & & 21 & 42 & & 67 & & 49 & 18 & 273 \\
\hline Cr Cajamarca & Ecuador & 14） & 114 & 128 & & 22 & 41 & & 65 & & 49 & 17 & 262 \\
\hline Cr Manabí & Ecuador & 16) & 132 & 167 & 135 & 22 & & 175 & 74 & 48 & 18 & & 456 \\
\hline CLT & México & 15) & 125 & 146 & 127 & 16 & 32 & 172 & & 46 & 17 & & 392 \\
\hline Cr PAT & Argentina & 17) & 123 & 159 & 125 & 25 & 54 & 170 & & 51 & & & \\
\hline Cr NOA & Argentina & 17) & 125 & 163 & 126 & 24 & 53 & 181 & & 53 & & & \\
\hline
\end{tabular}

${ }^{*}$ Cr PAT: Criollo Patagonia. Cr NOA: Criollo Noroeste de Argentina

${ }^{* * *}$ : : Bodisco y Pacheco (1962). 2: Ríos el al. (1959). 3: Jordão (1949). 4: Botero (1976). 5: Rubio. (1976). 6: Domingues (1961). 7: Pinzón (1952). 8: Jordão (1956). 9: Gracia (1947). 10: Ospina (1950). 12: Magofke y Bodisco (1966). 13: Bottani (2020). 14: Aguirre et al. (2011). 15: Canales (2014). 16: Cevallos et al. (2016). 17: Martínez (2008). 18: Contreras et al. (2011).

En el cuadro 5.1 a 5.3 se muestran los resultados de las correlaciones entre las medidas para el material en conjunto. Cuadro 5.1 Correlaciones entre medidas del conjunto.

\begin{tabular}{|c|c|c|c|c|c|c|c|c|c|c|c|}
\hline & $\begin{array}{l}\mathrm{AlCz} \\
(\mathrm{cm})\end{array}$ & $\begin{array}{r}\mathrm{AlGr} \\
(\mathrm{cm})\end{array}$ & $\begin{array}{l}\text { AlBC } \\
(\mathrm{cm})\end{array}$ & $\begin{array}{l}\text { PfPc } \\
(\mathrm{cm})\end{array}$ & $\begin{array}{r}\text { PmyE } \\
(\mathrm{cm})\end{array}$ & $\begin{array}{l}\text { PmnE } \\
(\mathrm{cm})\end{array}$ & $\begin{array}{l}\mathrm{AlCd} \\
(\mathrm{cm})\end{array}$ & $\begin{array}{r}\text { AlRd } \\
(\mathrm{cm})\end{array}$ & $\begin{array}{r}\mathrm{LnCp} \\
(\mathrm{cm})\end{array}$ & $\begin{array}{r}\mathrm{LnCa} \\
(\mathrm{cm})\end{array}$ & $\begin{array}{l}\mathrm{LnPv} \\
(\mathrm{cm})\end{array}$ \\
\hline$\overline{\text { Peso (kg) }}$ & $0.71^{* *}$ & $0.62^{* *}$ & $0.60^{* *}$ & $0.76^{* * *}$ & 0.10 & -0.12 & $0.43^{* *}$ & 0.16 & $0.61^{* * *}$ & $0.42^{* *}$ & $0.66^{* *}$ \\
\hline $\mathrm{AlCz}(\mathrm{cm})$ & & $0.79^{* *}$ & $0.73^{* *}$ & $0.74^{* *}$ & $0.44^{* *}$ & 0.1 & $0.61^{* *}$ & $0.37^{* *}$ & $0.58^{* *}$ & $0.41^{* *}$ & $0.61^{* *}$ \\
\hline $\operatorname{AlGr}(\mathrm{cm})$ & & & $0.85^{* *}$ & $0.65^{* *}$ & $0.46^{* *}$ & 0.16 & $0.60^{* *}$ & $0.44^{* *}$ & $0.49^{* *}$ & $0.39^{* *}$ & $0.58^{* *}$ \\
\hline $\operatorname{AlBC}(\mathrm{cm})$ & & & & $0.61^{* *}$ & $0.45^{* *}$ & 0.17 & $0.50^{* *}$ & $0.40^{* *}$ & $0.58^{* *}$ & $0.45^{* *}$ & $0.62^{* *}$ \\
\hline $\operatorname{PfPc}(\mathrm{cm})$ & & & & & 0.2 & -0.03 & $0.54^{* *}$ & $0.36^{* *}$ & $0.64^{* *}$ & $0.46^{* *}$ & $0.68^{* *}$ \\
\hline PmyE $(\mathrm{cm})$ & & & & & & $0.57^{* *}$ & $0.56^{* *}$ & $0.60^{* *}$ & $0.22^{*}$ & $0.24^{*}$ & $0.31^{* *}$ \\
\hline $\operatorname{PmnE}(\mathrm{cm})$ & & & & & & & $0.22^{*}$ & $0.31^{* *}$ & -0.07 & 0.11 & 0.01 \\
\hline $\operatorname{AlCd}(\mathrm{cm})$ & & & & & & & & $0.67^{* *}$ & $0.40^{* *}$ & $0.32^{* *}$ & $0.49^{* *}$ \\
\hline AlRd (cm) & & & & & & & & & $0.34^{* *}$ & $0.30^{* *}$ & $0.29^{* *}$ \\
\hline $\operatorname{LnCp}(\mathrm{cm})$ & & & & & & & & & & $0.45^{* *}$ & $0.64^{* *}$ \\
\hline $\mathrm{LnCa}(\mathrm{cm})$ & & & & & & & & & & & $0.49^{* *}$ \\
\hline
\end{tabular}

AlCz: Alzada a la cruz. AlGr: Alzada a la grupa. AlBC: Peso, Alzada a la base de la cola. PfPc: Profundidad del pecho. PmyE: Profundidad mayor al esternón. PmnE: Profundidad mínima al esternón. AlCd: Alzada al codo. AlRd: Alzada a la rodilla. LnCp: Longitud del cuerpo. LnCa: Longitud de la cabeza. LnPv: Longitud de la pelvis. AnCa: Ancho de la cabeza. AnPv: Ancho de la pelvis. AlGr: Alzada a la grupa. AnEs: Ancho de la espalda. AnPc: Ancho del pecho. EsPd: Espesor de la piel doblada. PrCñ: Perímetro de la caña. PrCr: Perímetro cardíaco. PrAb: Perímetro abdominal LnCn: Longitud del cuerno. PrCB: Perímetro del cuerno en la base. LnOj: Longitud de la oreja.

${ }^{*} \mathrm{p}<0.05 .{ }^{* *} \mathrm{p}<0.01$ 
Cuadro 5.2 Correlaciones entre medidas del conjunto (Continuación)

\begin{tabular}{|c|c|c|c|c|c|c|c|c|c|c|c|c|}
\hline & $\begin{array}{l}\mathrm{LnOj} \\
(\mathrm{cm})\end{array}$ & $\begin{array}{r}\mathrm{AnCa} \\
(\mathrm{cm}) \\
\end{array}$ & $\begin{array}{r}\mathrm{AnPv} \\
(\mathrm{cm})\end{array}$ & $\begin{array}{l}\text { AnGr } \\
(\mathrm{cm})\end{array}$ & $\begin{array}{r}\text { AnEs } \\
(\mathrm{cm})\end{array}$ & $\begin{array}{r}\text { AnPc } \\
(\mathrm{cm})\end{array}$ & $\begin{array}{r}\mathrm{EsPd} \\
(\mathrm{cm}) \\
\end{array}$ & $\begin{array}{r}\operatorname{PrC} \tilde{n} \\
(\mathrm{~cm})\end{array}$ & $\begin{array}{l}\mathrm{PrCr} \\
(\mathrm{cm})\end{array}$ & $\begin{array}{r}\operatorname{PrAb} \\
(\mathrm{cm})\end{array}$ & $\begin{array}{r}\mathrm{LnCn} \\
(\mathrm{cm})\end{array}$ & $\begin{array}{r}\operatorname{PrCB} \\
(\mathrm{cm})\end{array}$ \\
\hline Peso (kg) & 0.13 & $0.60^{* *}$ & $0.45^{* *}$ & $0.76^{* *}$ & $0.47^{* *}$ & $0.50^{* *}$ & $0.21^{*}$ & $0.63^{* *}$ & $0.74^{* *}$ & $0.65^{* *}$ & -0.12 & $0.24^{*}$ \\
\hline $\mathrm{AlCz}(\mathrm{cm})$ & $0.27^{*}$ & $0.47^{* *}$ & $0.42^{* *}$ & $0.67^{* *}$ & 0.17 & $0.21^{*}$ & $0.25^{*}$ & $0.60^{* *}$ & $0.65^{* *}$ & $0.50^{* *}$ & -0.14 & 0.16 \\
\hline $\operatorname{AlGr}(\mathrm{cm})$ & $0.36^{* *}$ & $0.47^{* *}$ & $0.43^{* *}$ & $0.60^{* *}$ & 0.18 & 0.18 & 0.12 & $0.45^{* *}$ & $0.58^{* *}$ & $0.41^{* *}$ & -0.06 & 0.18 \\
\hline $\mathrm{AlBC}(\mathrm{cm})$ & 0.19 & $0.45^{* *}$ & $0.39^{* *}$ & $0.61^{* *}$ & $0.22^{*}$ & $0.24^{*}$ & $0.21^{*}$ & $0.44^{* *}$ & $0.61^{* *}$ & $0.38 * *$ & -0.17 & 0.18 \\
\hline $\operatorname{PfPc}(\mathrm{cm})$ & 0.14 & $0.56^{* *}$ & $0.56^{* *}$ & $0.72^{* *}$ & $0.22^{*}$ & $0.29^{* *}$ & $0.26^{*}$ & $0.56^{* *}$ & $0.79^{* *}$ & $0.63^{* *}$ & -0.14 & 0.24 \\
\hline PmyE $(\mathrm{cm})$ & $0.26^{*}$ & 0.12 & 0.13 & 0.19 & -0.13 & $-0.21^{*}$ & 0.14 & -0.05 & 0.07 & -0.11 & 0.16 & $0.28 *$ \\
\hline PmnE $(\mathrm{cm})$ & 0.19 & 0.06 & 0.09 & -0.02 & $-0.21^{*}$ & $-0.37^{* *}$ & 0.02 & -0.18 & -0.13 & -0.15 & $0.30^{*}$ & $0.28 *$ \\
\hline $\operatorname{AlCd}(\mathrm{cm})$ & $0.28^{* *}$ & $0.27^{* *}$ & $0.34^{* *}$ & $0.41^{* *}$ & -0.01 & -0.01 & 0.14 & $0.24^{*}$ & $0.35^{* *}$ & $0.31^{* *}$ & 0.13 & $0.27^{*}$ \\
\hline $\operatorname{AlRd}(\mathrm{cm})$ & 0.19 & 0.2 & $0.23^{*}$ & 0.17 & $0.22^{*}$ & $0.21^{*}$ & 0.03 & 0.02 & $0.21^{*}$ & 0.03 & 0.16 & 0.15 \\
\hline $\operatorname{LnCp}(\mathrm{cm})$ & -0.4 & $0.38^{* *}$ & $0.36^{* *}$ & $0.57^{* *}$ & 0.13 & 0.14 & $0.32^{* *}$ & $0.54^{* *}$ & $0.50^{* *}$ & $0.39^{* *}$ & -0.08 & 0.16 \\
\hline
\end{tabular}

AlCz: Alzada a la cruz. AlGr: Alzada a la grupa. AlBC: Peso, Alzada a la base de la cola. PfPc: Profundidad del pecho. PmyE: Profundidad mayor al esternón. PmnE: Profundidad mínima al esternón. AlCd: Alzada al codo. AlRd: Alzada a la rodilla. LnCp: Longitud del cuerpo. LnCa: Longitud de la cabeza. LnPv: Longitud de la pelvis. AnCa: Ancho de la cabeza. AnPv: Ancho de la pelvis. AlGr: Alzada a la grupa. AnEs: Ancho de la espalda. AnPc: Ancho del pecho. EsPd: Espesor de la piel doblada. PrCñ: Perímetro de la caña. PrCr: Perímetro cardíaco. PrAb: Perímetro abdominal. LnCn: Longitud del cuerno. PrCB: Perímetro del cuerno en la base. LnOj: Longitud de la oreja.

${ }^{*} \mathrm{p}<0.05 .{ }^{* *} \mathrm{p}<0.01$

Cuadro 5.3 Correlaciones entre medidas del conjunto (Continuación)

\begin{tabular}{|c|c|c|c|c|c|c|c|c|c|c|c|c|}
\hline & $\begin{array}{c}\mathrm{LnOj} \\
\mathrm{n}=81\end{array}$ & $\begin{array}{c}\mathrm{AnCa} \\
(\mathrm{cm})\end{array}$ & $\begin{array}{l}\mathrm{AnPv} \\
(\mathrm{cm})\end{array}$ & $\begin{array}{r}\mathrm{AnGr} \\
(\mathrm{cm})\end{array}$ & $\begin{array}{c}\text { AnEs } \\
(\mathrm{cm})\end{array}$ & $\begin{array}{l}\mathrm{AnPc} \\
(\mathrm{cm})\end{array}$ & $\begin{array}{r}\mathrm{EsPd} \\
(\mathrm{cm})\end{array}$ & $\begin{array}{l}\operatorname{PrC\tilde {n}} \\
(\mathrm{cm})\end{array}$ & $\begin{array}{l}\mathrm{PrCr} \\
(\mathrm{cm})\end{array}$ & $\begin{array}{l}\text { PrAb } \\
(\mathrm{cm})\end{array}$ & $\begin{array}{c}\mathrm{LnCn} \\
\mathrm{n}=74\end{array}$ & $\begin{array}{l}\text { PrCB } \\
n=74\end{array}$ \\
\hline $\mathrm{LnCa}(\mathrm{cm})$ & 0.16 & $0.48^{* *}$ & $0.29^{* *}$ & $0.46^{* *}$ & 0.15 & 0.18 & -0.03 & $0.38^{* *}$ & $0.48^{* *}$ & $0.32^{* *}$ & -0.09 & 0.15 \\
\hline $\mathrm{LnPv}(\mathrm{cm})$ & 0.10 & $0.52^{* *}$ & $0.39^{* *}$ & $0.61^{* *}$ & 0.16 & 0.18 & $0.28^{* *}$ & $0.45^{* *}$ & $0.61^{* *}$ & $0.47^{* *}$ & -0.06 & 0.22 \\
\hline $\mathrm{LnOj}(\mathrm{cm})$ & & $0.25^{*}$ & $0.31^{* *}$ & $0.25^{*}$ & 0.06 & -0.03 & -0.02 & 0.16 & 0.15 & $0.22^{*}$ & 0.07 & 0.11 \\
\hline $\mathrm{AnCa}(\mathrm{cm})$ & & & $0.36^{* *}$ & $0.51^{* *}$ & $0.21^{*}$ & 0.18 & 0.18 & $0.49^{* *}$ & $0.53^{* *}$ & $0.45^{* *}$ & -0.17 & 0.15 \\
\hline $\operatorname{AnPv}(\mathrm{cm})$ & & & & $0.48^{* *}$ & $0.24^{*}$ & 0.19 & 0.14 & $0.34^{* *}$ & $0.49^{* *}$ & $0.37^{* *}$ & $-0.24^{*}$ & -0.04 \\
\hline AnGr (cm) & & & & & $0.48 * *$ & $0.46^{* *}$ & $0.27^{* *}$ & $0.56^{* *}$ & $0.69^{* *}$ & $0.59^{* *}$ & $-0.28^{*}$ & 0.17 \\
\hline AnEs (cm) & & & & & & $0.83^{* *}$ & 0.16 & $0.33^{* *}$ & $0.47^{* *}$ & $0.45^{* *}$ & $-0.39^{* *}$ & -0.09 \\
\hline $\operatorname{AnPc}(\mathrm{cm})$ & & & & & & & 0.16 & $0.35^{* *}$ & $0.56^{* *}$ & $0.54^{* *}$ & $-0.31^{* *}$ & -0.10 \\
\hline $\operatorname{EsPd}(\mathrm{cm})$ & & & & & & & & $0.22^{*}$ & 0.08 & 0.14 & -0.09 & $0.25^{*}$ \\
\hline $\operatorname{PrCñ}(\mathrm{cm})$ & & & & & & & & & $0.57^{* *}$ & $0.49^{* *}$ & -0.06 & $0.26^{*}$ \\
\hline $\operatorname{PrCr}(\mathrm{cm})$ & & & & & & & & & & $0.67^{* *}$ & $-0.25^{*}$ & 0.03 \\
\hline $\operatorname{PrAb}(\mathrm{cm})$ & & & & & & & & & & & -0.15 & 0.13 \\
\hline $\mathrm{LnCn}(\mathrm{cm})$ & & & & & & & & & & & & $0.59^{* *}$ \\
\hline
\end{tabular}

AlCz: Alzada a la cruz. AlGr: Alzada a la grupa. AlBC: Peso, Alzada a la base de la cola. PfPc: Profundidad del pecho. PmyE: Profundidad mayor al esternón. PmnE: Profundidad mínima al esternón. AlCd: Alzada al codo. AlRd: Alzada a la rodilla. LnCp: Longitud del cuerpo. LnCa: Longitud de la cabeza. LnPv: Longitud de la pelvis. AnCa: Ancho de la cabeza. AnPv: Ancho de la pelvis. AlGr: Alzada a la grupa. AnEs: Ancho de la espalda. AnPc: Ancho del pecho. EsPd: Espesor de la piel doblada. PrCñ: Perímetro de la caña. PrCr: Perímetro cardíaco. PrAb: Perímetro abdominal. LnCn: Longitud del cuerno. PrCB: Perímetro del cuerno en la base. LnOj: Longitud de la oreja.

${ }^{*} \mathrm{p}<0.05 .{ }^{* *} \mathrm{p}<0.01$

Los coeficientes de correlación de Pearson conseguidos entre las variables fluctúan en cuanto a magnitud y nivel de significación para las diferentes líneas y para el total. Sin embargo, las correlaciones entre variables de importancia económica, como aquellas que incluyen Peso, Alzada a la cruz, Profundidad del pecho, Longitud del cuerpo y Perímetro cardíaco dieron resultados positivos y significativos, siempre mayores en el CrRL que en el CrLl.

\section{Conclusiones}

Los valores de correlación obtenidos para ambas poblaciones en conjunto que se muestran en el Cuadro 5.1 a 5.3 ofrecen un grado de armonía moderado (Cevallos el al., 2016) pues en la generalidad de las dimensiones estructurales, el $65 \%$ de los coeficientes resultaron positivos y significativos ( $\mathrm{p}<0.05$ ), siendo bajas o inexistentes las correlaciones con Longitud de la oreja, Longitud y Perímetro del cuerno, así como ambas Amplitudes al esternón. Es de hacer notar, que las pocas correlaciones de signo negativo resultaron no significativas ( $\mathrm{p}>0.05$ ) en su totalidad.

Los valores de desviación típica y los coeficientes de variación indicaron que la variabilidad fenotípica del CrRL era ligeramente menor a la del CrLl. pero ambas comparables a otros criollos latinoamericanos.
Las vacas CrRL superaron al CrLl en las dimensiones más importantes. El peso, $20 \%$ superior encabezó la lista, mientras que en las demás dimensiones la ventaja osciló entre 3 y $8 \%$ a favor del CrRL. 
La comparación de las medidas bovino-métricas de mayor interés en el Criollo Latinoamericano puso en evidencia la similitud entre las poblaciones estudiadas, tratándose en general de animales pequeños, equilibrados con los recursos disponibles.

Intentar comparar resultados obtenidos en vacas de edad o número de parto, estado de lactación o de preñez, estación, dieta y manejo distintos, realizado por equipos de investigadores en lugares, instalaciones, métodos e instrumentos también diferentes, resultó marcadamente complejo.

Las correlaciones entre variables de importancia económica como aquellas que incluyen Peso, Alzada a la cruz, Profundidad del pecho, Longitud del cuerpo y Perímetro cardíaco dieron resultados positivos y significativos, siempre mayores en el CrRL que en el CrLl.

En cuanto a su porvenir, quizás sea demasiado tarde para recomendar la conservación y valoración de estas dos poblaciones cuya contribución a la variabilidad genética necesaria para acoplar genotipos específicos con diferentes sistemas de cría y condiciones ambientales alteradas es inobjetable.
Las estrategias implementadas por el Estado (FlorioLuis y Pineda-Graterol, 2018) no han sido suficientes ni efectivas para la preservación ni aprovechamiento de ambas poblaciones. De hecho, el esfuerzo realizado para reunir un rebaño $\mathrm{CrLl}$ en Calabozo fue comprometido al adoptar un plan de apareamiento de absorción con Brahman y Criollo Rio Limón.

El demérito de las poblaciones locales se origina en la prerrogativa otorgada a la producción y el ingreso sobre el desempeño económico y el costo, cuando la discusión sobre sistemas de producción sin considerar los resultados económicos es irrelevante (Madalena 2002).

El Sistema Científico y Tecnológico venezolano consciente de que estas poblaciones autóctonas suponen una fuente de información genética interesante y poco conocida (Aranguren et al., 2011) está en capacidad de realizar estudios de caracterización molecular de estas razas y su potencial en la producción animal con el objeto de promover la utilización de este germoplasma en los sistemas de ganadería comercial del país.

\section{Conflicto de intereses}

El autor declara no tener conflicto de intereses de ningún tipo.

\section{Reconocimiento}

Al Perito Agropecuario Santiago Borsotti por su colaboración en la medición de los animales.

\section{Lista de fuentes de financiación}

Esta investigación no recibió ninguna subvención específica de agencias de financiación en los sectores público, comercial o sin fines de lucro. Los costos incurridos fueron sufragados en el presupuesto ordinario de la Estación Experimental de Los Llanos, MAC y la Facultad de Ciencias Veterinarias de la UCV.

\section{Literatura citada}

Abreu, O., S. Labbe y M. Perozo. 1977. El ganado Criollo venezolano en la producción de leche y carne. FONAIAP-CIARZU. Bol. Téc. №1. 77 p.

Aguirre, L., Bermeo, A., Maza, D. \& Merino, L. 2011. Estudio fenotípico y zoométrico del bovino criollo de la sierra media y alta de la región sur del Ecuador (RSE). Actas Iberoamericanas de Conservación Animal 1: 392-397.

Aranguren-Méndez, J. A., Portillo, M. G., Yáñez, L. F., Rincón, X., Contreras, G. y Villasmil-Ontiveros, Y. 2011. Caracterización genética del ganado criollo limonero a través de genes de interés productivo. AICA 1 (2011) 199-202.

Bodisco, V. y Abreu, O. 1981. Producción de leche por vacas criollas puras. En: Estudio FAO, Producción y Sanidad Animal. 22: 17-39.
Bodisco, V. Y J.J. Pacheco. 1962. Peso de las vacas Criollas y Pardo Suizas en el Centro de Investigaciones Agronómicas. III Jornadas Agronómicas, Cagua, Venezuela (Mimeógrafo) 14 PP.

Botero, F.M. 1976. Ganado Blanco Orejinegro. En Razas Criollas Colombianas. Instituto Colombiano Agropecuario. Bogotá, Colombia. Manual de Asistencia Técnica 21:17-61.

Bottani, Gabriela (2020). Bolivian creole cattle: population structure, genetic diversity and management practices. Diss. (summary) Uppsala: Sveriges lantbruksuniv. Acta Universitatis Agriculturae Sueciae, 1652-6880; 2020:7 [Doctoral thesis] 
Bracho M., I. E., Contreras M., G. E.; Pirela L., M. F. y Zambrano N., S. A. 2002. Capítulo I. La raza criollo limonero: una realidad para la ganadería de doble propósito. En Avances en la ganadería de doble propósito. Trujillo, octubre de 2002. (Editores: Ramírez I., Lílido N.; González Stagnaro, Carlos y Soto Belloso, Eleazar).

Canales Vergara, A. M. 2014. Caracterización genética y morfológica de vacas de la raza Criollo Lechero Tropical. Tesis Maestro en Ciencia Animal, Facultad de Medicina Veterinaria y Zootecnia, Universidad Veracruzana, Veracruz, México.

Cevallos F., O. F. 2012. Caracterización morfoestructural y faneróptico del bovino criollo en la provincia de Manabí, Ecuador. Trabajo de fin de Master Universidad de Córdoba, Facultad de Veterinaria, Departamento de producción animal, Master en zootecnia y gestión sostenible: ganadería Ecológica e integrada. 67 PP.

Cevallos F., O.F., Cecilio Barba, C., Delgado, J. V., González, A., Perea, J., Angón, E. y Antón García, A. 2016. Caracterización zoométrica y morfológica del ganado criollo de Manabí (Ecuador). Revista Científica, FCV-LUZ / Vol. XXVI (5): 313-323.

Contreras G, Chirinos Z, Zambrano S, Molero E, Páez A. 2011. Caracterización morfológica e índice zoométrico de vacas criollo limonero de Venezuela. Rev. Fac. Agron. 28: 91-103.

Fisher, R. A. 1925. Statistical Methods for Research Workers. 239 pp., Edinburgh and London

Florio-Luis, J. y Pineda-Graterol M. 2018. Situación actual en la preservación del bovino criollo limonero en la República Bolivariana de Venezuela. AICA 12 (2018) 133-140.

Frómeta, L., D. Plasse, M. González, C. Chicco, B. Muller-Haye, E. Cevallos y N. Pena De Borsotti. 1974. Comportamiento productivo de Bos taurus y Bos índicus y sus cruces. IV. Crecimiento hasta 18 meses. ALPA Memoria 9:48 (Resumen).

Gandini, G. C., and Villa, E. 2003. Analysis of the cultural value of local livestock breeds: A methodology. J. Anim. Breed. Genet. 120, 1-11. doi:10.1046/j.1439-0388.2003.00365.x

Gracia, C E. 1947. El ganado Blanco Orejinegro. Agricultura Tropical 3(11): 17-21.

Jordão, L.P. 1949. Alguns dados sobre La raça Caracu. Revista Rural Brasileira 29(349): 78-83.

Jordão, L.P. 1956. Estudo retrospectivo e comparativo de dados sobre bovinos de raças Caracu y Mocha Nacional. Boletim de Indústria Animal 15: 123-138.

Linares, T., D. Plasse, M. Burguera, J. Ordóñez, J. Ríos, O. Verde y M. González. 1974 a. Comportamiento productivo en Bos taurus y Bos índicus y sus cruces en el Llano Venezolano. I. Eficiencia reproductiva. ALPA Memoria 9:289-301.
Linares, T., M. Burguera, D. Plasse, J. Ordóñez, O. Verde, M. González Y L. Frómeta.1974b. Comportamiento productivo de Bos Taurus y Bos índicus y sus cruces. V. Pubertad en novillas. ALPA Memoria 9:91 (Resumen).

Lush, J. L. \& Copeland, O. C. (1930) A study of the accuracy of measurements of dairy cattle. J. Agric. Res. 41(1):37-49

Madalena, F. E. 2002. Cruces entre razas bovinas para producción económica de leche. Memorias XI Congreso Venezolano de Producción e Industria Animal. Valera ULA-Trujillo. http://www.fernandomadalena.com/site_arquivos/ 007.pdf

Magofke, S. y V. Bodisco. 1966. Estimaciones del mejoramiento genético del ganado Criollo lechero en Maracay, Venezuela, entre los años 1955-64. ALPA Memoria 1:105-127

Martínez, R. D. (2008). Caracterización genética y morfológica del bovino criollo Argentino de origen patagónico. Universidad Politécnica de Valencia, Departamento de Ciencia Animal, Tesis Doctoral. Valencia. $244 \quad$ pp. http://www.uco.es/conbiand/tesis/Ruen_Martinez. pdf

Ordóñez V., J. A. 2020. Further consideration on mating systems for the tropics. NOVASINERGIA 3 (1):6-16. Doi: 10.37135/ns.01.05.01

Ordóñez V., J. A., T. Linares, D. Plasse, O. Verde, M. Burguera y R. Gil. 1974. Comportamiento productivo de Bos taurus y Bos índicus y sus cruces. VI. Estimación de heterosis en edad y peso a pubertad en novillas. ALPA Memoria 9:90 (Resumen).

Ospina, A. 1950. Características del ganado Blanco Orejinegro. Agricultura Tropical (Bogotá) 6 (2):12-19.

Peña De Borsotti, N., B. Muller-Haye, O. Verde, D. Plasse, D. Ríos y M. González. 1974. Comportamiento productivo de Bos taurus y Bos índicus y sus cruces. II. Peso al nacer. ALPA Memoria 9:46-47 (Resumen).

Pinzón, E. 1952. Razas Criollas Venezolanas. Agricultura Tropical 8(1):15-21; 8(2):9-15.

Plasse, D. 1981 El uso de1 ganado criollo en programas de cruzamiento para la producción de carne en América Latina. Estudio FAO: Producción y Sanidad Animal 22: 77-107. http://www.fao.org/3/ah223s/AH223So8.htm\#ch4 .7

Plasse, D., L. Frómeta, B. Ríos, M. González, R. Gil, E. Cevallos y N. Pena De Borsotti.1974a. Comportamiento productivo de Bos taurus y Bos índicus y sus cruces. III. Crecimiento predestete. ALPA Memoria 9:47-48 (Resumen). 
Plasse, D., O. Verde, B. Muller-Haye, M. Burguera y J. Ríos. 1974b. Comportamiento productivo de Bos taurus y Bos índicus y sus cruces. VII Estimación de heterosis en crecimiento. ALPA Memoria 9:61-62 (Resumen).

Ríos, C.E., V. Bodisco y F.J. Morillo. 1959. Selección del Ganado Criollo Lechero en Venezuela. Centro de Investigaciones Agronómicas, Maracay. Mimeógrafo, 35 p. citado por Bodisco, y Abreu (1981).
Rodríguez-Viso, M. Sin fecha. Guías de Zootecnia General. Cátedra de Zootecnia, FCV-UCV, Venezuela. Mimeo 4 PP.

Rouse, J. E. 1977. The criollo: Spanish cattle in the Americas. Norman, USA; University of Oklahoma Press. 303 pp.

Rubio, R. 1976. Ganado Costeño con Cuernos. Razas criollas colombianas. Manual de Asistencia Técnica. ICA (Col). No. 21: 83-106. 